\title{
Robotic approach for mediastinal diseases: state-of-the-art and current perspectives
}

\author{
Tugba Cosgun ${ }^{1}$, Erkan Kaba ${ }^{1}$, Alper Toker $^{2}$ \\ ${ }^{1}$ Department of Thoracic Surgery, Istanbul Bilim University, Istanbul, Turkey; ${ }^{2}$ Department of Thoracic Surgery, Istanbul University, Istanbul, \\ Turkey \\ Contributions: (I) Conception and design: T Cosgun, A Toker; (II) Administrative support: E Kaba; (III) Provision of study materials or patients: \\ None; (IV) Collection and assembly of data: T Cosgun; (V) Data analysis and interpretation: None; (VI) Manuscript writing: All authors; (VII) Final \\ approval of manuscript: All authors. \\ Correspondence to: Tugba Cosgun. Sisli Merkez Mah., Abide-i Hurriyet Cad. No. 164, Sisli, Istanbul, Turkey. Email: tugba_Cosgun@hotmail.com.
}

\begin{abstract}
In the past, mediastinal surgery required a sternotomy approach to maximize exposure. Subsequently, in the 1990s, minimally invasive techniques, including thoracic surgery, became a popular matter of debate in many surgical specialties. Video-assisted thoracic surgical techniques have several advantages over an open approach, including less trauma, shorter hospitalization duration, better cosmetic results, and preserved lung function. It also presents disadvantages such as less surgical exposure and inadequate technical requirements. In this review, we analyzed current literature and authors' expertise in robot-assisted thoracic surgery, which provides better flexibility, more intuitive movements, and highdefinition and three-dimensional view, in mediastinal surgeries.
\end{abstract}

Keywords: Mediastinal tumor; open surgery; video-assisted surgery; robot-assisted thoracoscopic surgery (RATS)

Received: 07 June 2018; Accepted: 08 November 2018; Published: 04 December 2018.

doi: $10.21037 /$ shc.2018.11.04

View this article at: http://dx.doi.org/10.21037/shc.2018.11.04

\section{Introduction}

Complete surgical resection of mediastinal tumors requires excellent exposure. Large tumors in proximity to the vital structures require high level of expertise in the dissection techniques and sufficient knowledge for weighing risks and benefits during certain surgical steps. Open surgical techniques include thoracotomy, sternotomy, clamshell/ hemiclamshell, partial sternal splitting incisions, or a combination of these. If carefully selected, open surgery provides the best exposure in the mediastinum. However, open surgery has a higher mortality, morbidity, long hospitalization, and increased bleeding compared with the minimally invasive techniques. We believe that open surgery should be specifically reserved for highly complex mediastinal tumors, such as invasive tumors in the mediastinum that require superior vena cava superior resection, major lung resection, or another vital organ resection in the thoracic cavity (Figure 1).
Video-assisted thoracoscopic surgery (VATS) was popularized in the late 1990s and early 2000s for lung resections and thymectomy for myasthenia gravis (MG). It aimed to decrease the morbidity, mortality, hospitalization duration, and several cosmetic incisions. This surgical experience with VATS for thymectomy in MG was implemented in small thymoma surgeries $(1,2)$. Subsequently, posterior mediastinal tumors such as neurogenic tumors were also included as ideal candidates for resection by VATS. In the last decade, with increased experience in VATS and development in technology, robotassisted thoracoscopic surgery (RATS) became available in some thoracic surgery centers. Currently, anterior and middle mediastinal tumors are recognized as ideal candidates for minimally invasive resection. However, there is no consensus among surgeons regarding the best minimally invasive technique between VATS and RATS. RATS provides better exposure owing to high-definition, three-dimensional view and retracting capabilities of the 

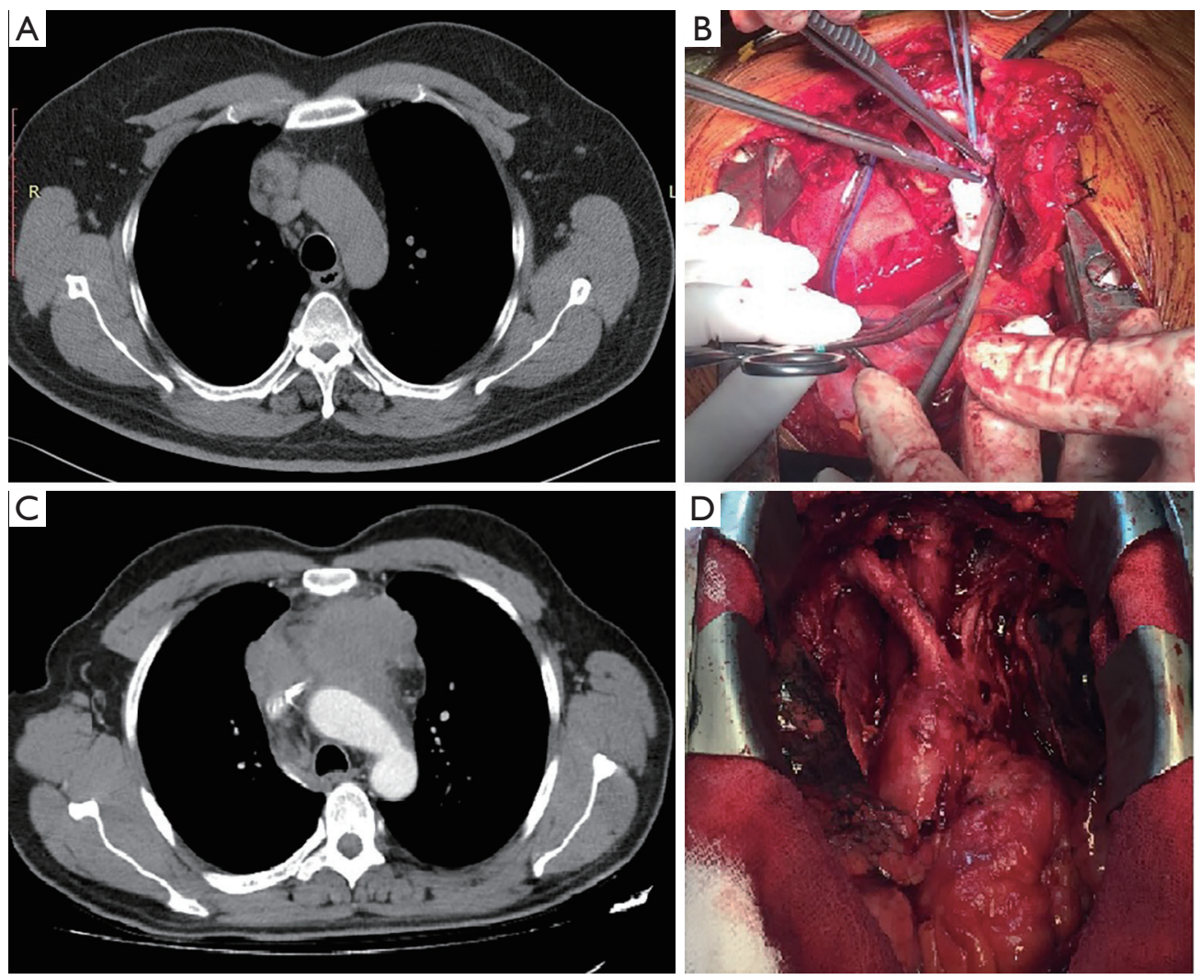

Figure 1 Patients with open mediastinal mass resections. (A) A hemangioendothelioma originating from the wall of vena cava could be seen in chest CT; (B) operative view, superior vena cava replacement; (C) a thymoma patients with an invasive thymoma after neoadjuvant chemotherapy treatment; (D) operative view of same patient.

robot's fourth arm. Besides, it provides better flexibility because of endo-wrist instruments, thus enabling more intuitive movements. These intraoperative advantages of minimally invasive techniques with RATS claimed lower mortality and morbidity, better quality of life (QOL), and shorter hospitalization duration (1). In this review, we explain the role and technical details of robotic surgical techniques for mediastinal tumors along with intraoperative tips and tricks.

\section{Anterior mediastinum}

The most important benefits of minimally invasive thymic resections are better cosmetics incisions and shorter hospitalization durations. Its additional benefits include a shorter duration of surgery and postoperative pleural drainage and decreased blood loss (3). Another study analyzing the outcomes of an open trans-sternal incision and VATS thymectomy for thymoma resection demonstrated that patients who underwent VATS thymectomy had fewer complications compared to open surgery (4). In addition to the early postoperative advantages, midterm results demonstrated that VATS thymectomy had oncological outcomes comparable with trans-sternal thymectomy regarding the adjuvant therapy received, disease progression, and survival. The midterm results of minimally invasive thymoma resections have also been reported to be reproducible (4,5). Moreover, following minimally invasive thymoma resections, major complications such as sternotomy dehiscence or infection are infrequent.

After resection of mediastinal tumors by VATS gained popularity and the indications and demand of using VATS for thymoma operations expanded, a requirement for developing terms, definitions, and policies for minimally invasive procedures became apparent. These were developed by the International Thymic Malignancy Interest Group (ITMIG) (6). Based on the existing literature, surgeons with experience in minimally invasive thymic resections specified 
standardized definitions, terms, and procedures for such resections. We believe that the outcome of the meeting of ITMIG improved the quality of minimally invasive thymoma resections as well as open thymoma surgery.

\section{RATS thymectomy for $M G$}

Thymectomy has been considered as an important component in the treatment of non-thymomatous MG. This theory was recently proven with a prospective randomized trial in patients with non-thymomatous MG who were under prednisone therapy (7). An extended transsternal thymectomy was the preferred procedure in this trial owing to its reproducibility and safety. The surgical therapy was combined with a standardized prednisone treatment. Furthermore, the researchers aimed to evaluate if the inclusion of surgery provides a better health status (decreased myasthenic weakness, administration of prednisone when needed, and improved QOL) compared with prednisone therapy alone after 3 years (7). This randomized, medication-controlled, rater-blinded trial demonstrated superior clinical outcomes in the surgical arm than in the prednisone therapy alone with regard to the need for prednisone and azathioprine, distress level due to immunosuppressive agents, and need for hospitalization due to exacerbations (7).

In the last two decades, the development of minimally invasive surgery has led to an improved acceptance of thymectomy by both neurologists and patients alike. This is because of an adequate tissue exposure provided by the new VATS techniques and decreased morbidity and mortality. The introduction of the da Vinci robotic system (Intuitive Surgical, Inc., Sunnyvale, CA, USA) has been considered as a further step in minimally invasive radical thymectomy. The ergonomic benefits of this system in mediastinal surgeries have previously been described (8). In addition, robotic surgical procedures have been reported to have a shorter learning curve (9).

Most of the surgical experience with RATS in mediastinal diseases is associated with thymic surgery for MG. Recently, for thymectomy and thymoma resection, RATS has been accepted as an alternative approach to open surgery or VATS (10). However, a radical thymectomy with the lowest degree of invasiveness is essential for patients with MG. To evaluate the possible advantages of RATS thymectomy over VATS thymectomy, the Berlin Group designed a retrospective study (11) where no age, MG severity, or pathological tissue (rate of the hyperplastic thymus) differences were noted. The cumulative complete remission rate of MG was reported to be $39.25 \%$ using RATS and $20.3 \%$ using VATS (11). The authors concluded that resection with robotic surgery provided a better response (11) and RATS thymectomy in patients with MG was safe and feasible (11). Other studies using RATS also demonstrated neurological benefit and a decreased need for corticosteroid therapy (12).

Other studies focused on the early postoperative outcomes between RATS and other alternatives. A study that compared RATS and VATS thymectomy did not report any statistical difference in conversion to open, hospitalization duration, or postoperative pneumonia between the two groups (13). A meta-analysis found no significant differences on conversion rates, operation time and length of hospitalization. Just a slightly higher blood loss was determined in the RATS group (14).

\section{Anterior mediastinal tumor resections}

Here, we compare the resection of anterior mediastinal lesions, such as mediastinal cysts, thymoma, thymic carcinoma, thymic hyperplasia, and liposarcoma, performed using different conventional methods. Seong et al. (15) compared sternotomy and RATS and found better early outcomes, such as shorter chest-tube drainage time, reduced blood loss, and shorter hospitalization duration (15). Another study on patients with thymoma, thymic cysts, thymic hyperplasia, metastasis of primary tumors, or extragonadal germ cell tumor compared the QOL of patients following RATS and open surgery (16). It demonstrated that QOL was better with RATS and recommended the use of robotic surgery for anterior mediastinal lesions $<4 \mathrm{~cm}$ (16) (Figure 2).

\section{Technical variations in robotic surgery for anterior mediastinal surgery}

With respect to technical approaches, authors have different preferences. Some surgeons prefer the right side whereas others prefer the left side of the hemi-thoracic cavity. To address this issue, high-quality subxiphoid RATS thymectomy resection has been developed, which enables adequate visualization of the phrenic nerves on both the sides (17).

While performing RATS for a standard thymectomy, we use three arms from the right side. However, we prefer the right-side approach because of the extra room to maneuver 

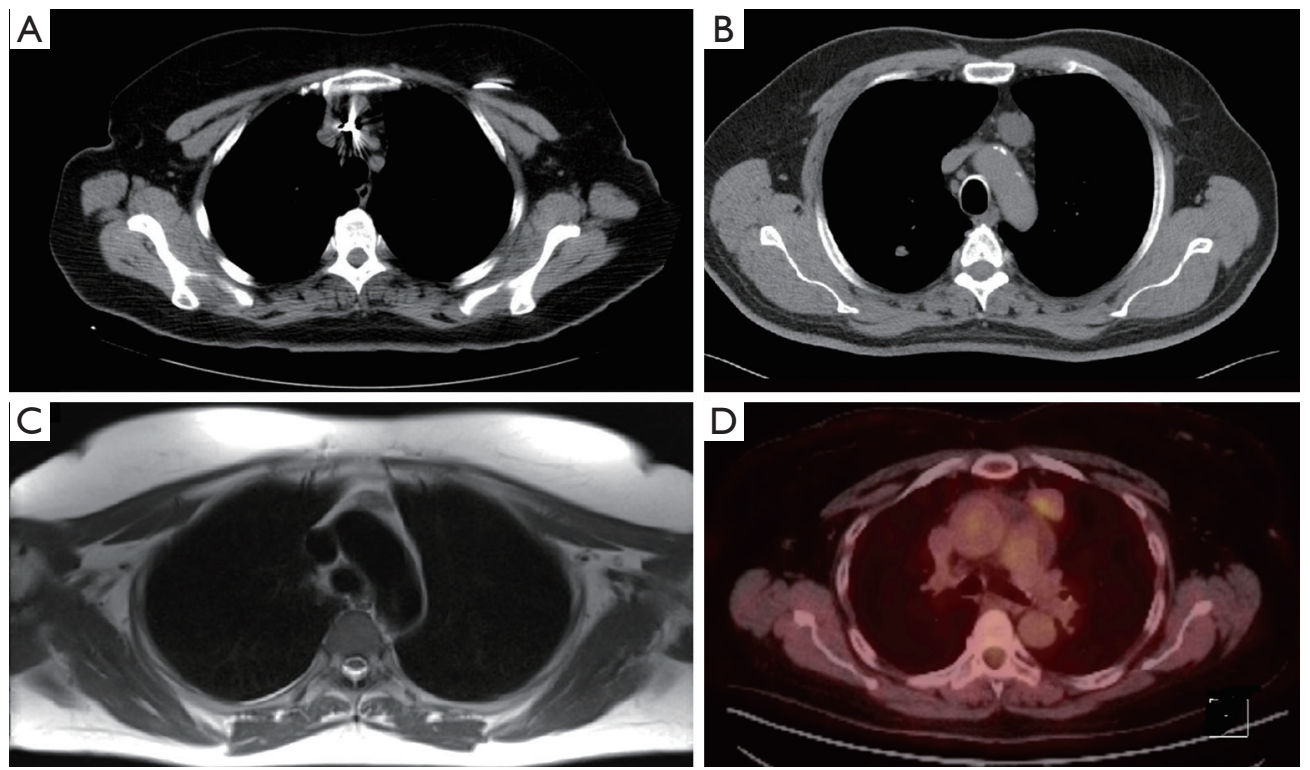

Figure 2 Images of patients who had robotic thymoma resection. (A) Computed tomography scan of a patient with thymoma; (B) a 2.5 cm of thymoma; (C) magnetic resonance imaging of a patient with $2 \mathrm{~cm}$ of thymoma; (D) positron emission tomography of a patients with 3 FDG uptake.

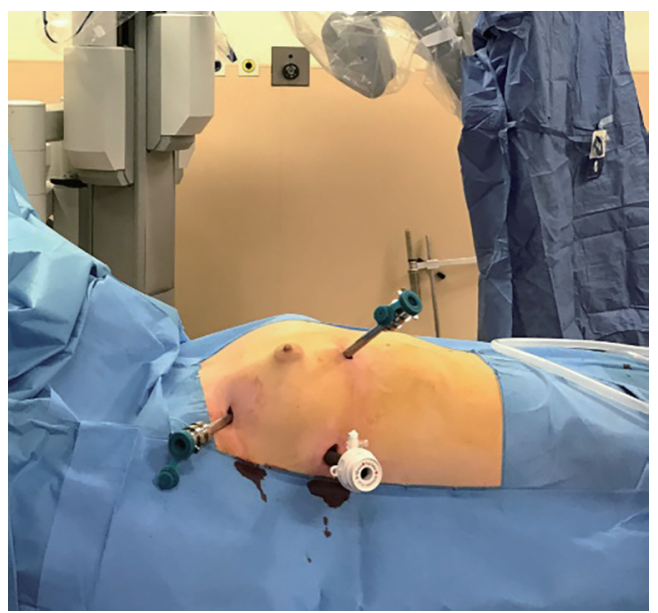

Figure 3 Placement of ports in standard thymectomy operation in myasthenia gravis.

surgical instruments and clearer visibility of landmarks. Thus, we also believe that the right-side approach is advantageous even if a thymoma is located on the left side (18). For a standard thymectomy for MG, we use three ports without the assistant port (Figure 3).

We consider that a surgeon's presence at the table is not essential in such cases; however, a surgeon is still assigned for the surgery in cases of emergency. Previously, a four- port technique was preferred for RATS thymectomy; however, we believe this approach can increase the pain compared with VATS surgery with two or three ports and RATS with three ports. We use $\mathrm{CO}_{2}$ insufflation in all patients; it prevents small bleedings and enlarges the space providing better exposure. An insufflation pressure of $8 \mathrm{mmHg}$ is maintained. All three camera modes are preferred: $30^{\circ}$ up, $0^{\circ}$, and $30^{\circ}$ down. In our technique, dissection is performed from the right-side peri cardiophrenic tissue, followed by the separation of the right thymus from the pericardium and superior vena cava until the right upper pole of the thymus is pulled down (Figure 4). At this point, $\mathrm{CO}_{2}$ insufflation is discontinued and the contralateral pleura under the sternum is opened. Subsequently, the left, lowerpole peri cardiophrenic fatty tissue is dissected, and the left upper pole is pulled until the thymic vein/s become visible. These veins are generally clipped (Figure 5).

Other surgeons such as Mineo et al. (21) and Pardolesi et al. (22) prefer the left-sided approach. Studies have demonstrated that these techniques are comparable with respect to surgical time, intraoperative blood loss, hospitalization duration, and postoperative complications (23). Subxiphoid incision is a recent method that has been defined as safe and feasible with good cosmetic and shortterm outcomes (17). Additionally, it avoids injuries to the 


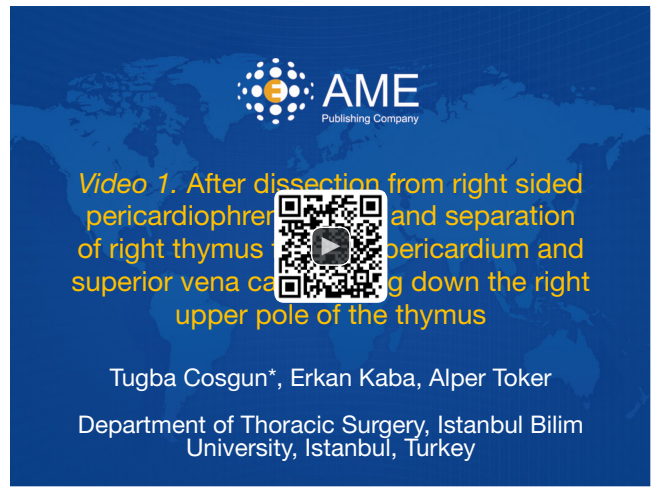

Figure 4 After dissection from right sided pericardiophrenic tissue and separation of right thymus from the pericardium and superior vena cava, pulling down the right upper pole of the thymus (19). Available online: http://www.asvide.com/article/view/28631

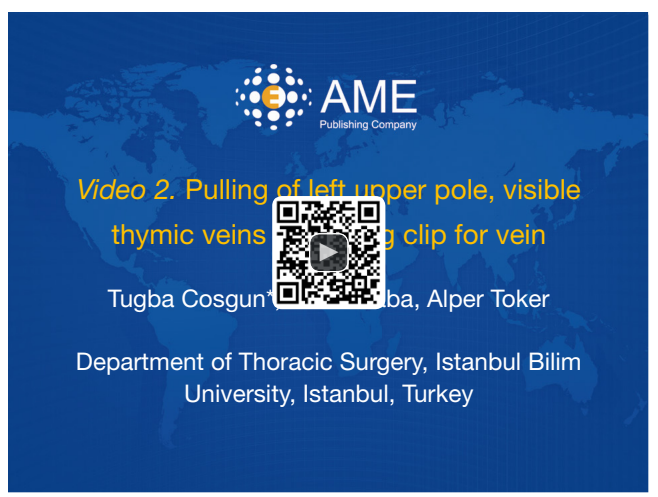

Figure 5 Pulling of left upper pole, visible thymic veins and using clip for vein (20).

Available online: http://www.asvide.com/article/view/28632

intercostal nerves, which can also reduce postoperative pain and chronic post-thoracotomy neuralgia (24). In a series of 17 patients, Weaver reported that the mean operation time was $2.5 \mathrm{~h}$ and mortality was $0 \%$ at postoperative day 30 (25).

The right-side and subxiphoid VATS with double sternum elevation (26) is another surgical technique for thymectomy. It involves a subxiphoid part wherein a 4- to $6-\mathrm{cm}$ transverse incision is made on the xiphoid process. Selective left-lung and right-lung ventilations are used. A sternal retractor is then placed under the sternum, which elevates it and facilitates access to the anterior mediastinum from below. A thoracoscopic port from the right side facilitates dissection. After dissection of the mediastinal tissue, a single-tooth hook is inserted to manubrium percutaneously to improve exposure to the superior mediastinum and lower neck regions (26). Robotic subxiphoid thymoma resection technique is a partial reproduction of this surgical technique.

Trans-subxiphoid thoracoscopic thymectomy has been reported to provide excellent visualization of the thymus and bilateral phrenic nerves (27). In this approach, an extended thymectomy with adequate bilateral mediastinal fatty tissue resection is performed. The technique includes a $2-\mathrm{cm}$, longitudinal skin incision for camera insertion below the xiphoid process. $\mathrm{CO}_{2}$ is insufflated via the subxiphoid camera-access incision. The lower mediastinal pleura is separated from behind the sternum, which is followed by making two holes below the bilateral costal arches. The bilateral mediastinal pleura, thymus, and peri-thymic fatty tissue are removed along with the direction of the phrenic nerve until the neck by pulling down the horns of the thymus.

\section{Upper borders in robotic thymoma resections}

Evaluation of upper limits in minimally invasive thymoma resection using RATS is essential to understand the capabilities of robotic surgeries. The outcomes of the surgery are dependent on the size or invasiveness of the tumor to the vital structures, which is determined using Masaoka-Koga staging. In a previous report, we demonstrated that Masaoka stage I and II tumors can be safely and efficiently resected using VATS (28). In our experience, as the tumor stage increases, a tendency for conversion to open surgery, postoperative hospitalization duration and readmissions increases (28). Additionally, we demonstrated that the learning curve is steep, and a certain level of expertise is essential in minimally invasive resection of a thymoma using VATS (2). Moreover, we did found no correlation between the size of the tumor and the stage of the thymoma (2).

Prior to selecting an ideal patient for robotic surgery, their radiographic findings should be analyzed. Invasion to the lung, major vessels, phrenic or recurrent laryngeal nerves could be considered as indicators for an open surgery. According to the ITMIG definitions, the involvement of the phrenic nerve, innominate vein, or other major vessels is a definitive contraindication to perform a minimally invasive surgery. However, we believe that phrenic nerve invasion alone, if found intraoperatively, can be managed using minimally invasive approaches. In such a case, we recommend diaphragmatic plication during the 


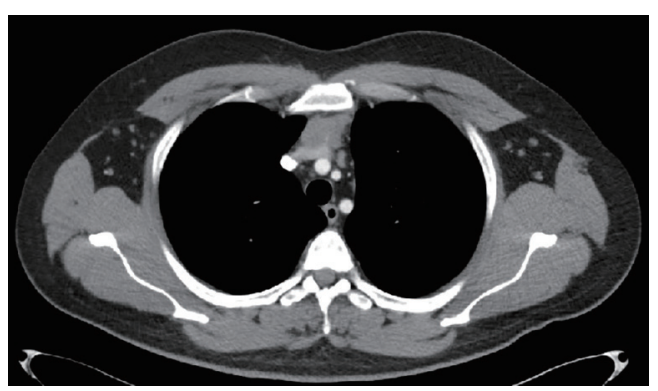

Figure 6 A patient with non-seminomatous germ cell tumor. After neoadjuvant chemotherapy, the mass was shrinked. It is still invading the left innominate vein. The surgery was performed with RATS. Operating surgeon contributed while stapling of left innominate vein from proximal and distal of the left innominate vein. RATS, robot-assisted thoracoscopic surgery.

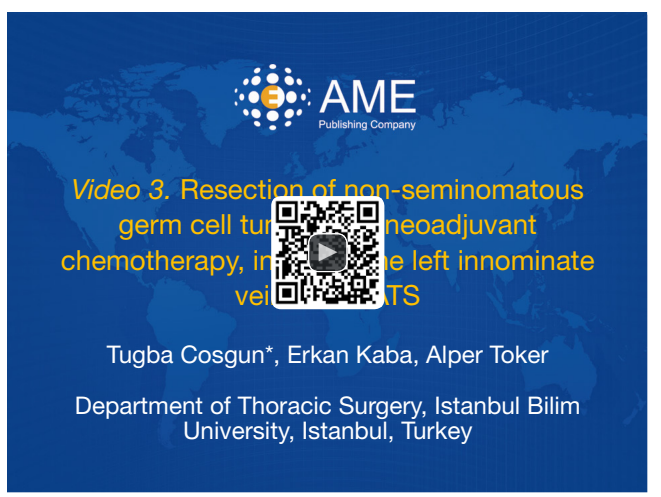

Figure 7 Resection of non-seminomatous germ cell tumor after neoadjuvant chemotherapy, invading the left innominate vein with RATS (30). Operating surgeons' contribution while stapling of left innominate vein.

Available online: http://www.asvide.com/article/view/28633

same surgery in case the phrenic nerve is sacrificed. Several highly experienced VATS surgeons have demonstrated VATS resections for Masaoka stage III and IVa tumors (29). In this study, out of the 77 patients with thymomas $<5 \mathrm{~cm}$, 13 presented with invasive thymomas (Masaoka stage III and IV). Their experience included limited resection of the phrenic nerve, pericardium, peri-thymic fat, and lung wedge. Even if these are stage III and IV thymomas, it can be concluded that these patients have been properly selected and it could be difficult to reproduce a similar experience on all patients with the same characteristics. The mean size of the thymomas in all patients was $34 \mathrm{~mm}$ (range, $23-55 \mathrm{~mm}$ ). All the patients underwent adjuvant radiotherapy and were followed up for 4.9 years (range, 1-10 years); only one case of local recurrence was reported (29).

It should be taken into consideration that the invasiveness of thymoma does not always correlate with its size. Rather, it is the stage that indicates the feasibility of minimally invasive resection. However, a shift to open surgery should be considered wisely because any pathological or surgical issue can compromise the procedure (6). Sometimes hybrid procedures combining RATS with VATS may be required in case of tumor invasion to major vessels, e.g., cases wherein patients with non-seminomatous germ cell tumor were operated after chemotherapy. A surgeon's intervention at the surgical table may be required to staple the innominate vein (Figures 6,7).

The dimension of the tumor is also important in the use of RATS. The appropriate size of thymoma for proceeding with a minimally invasive surgery is still a subject of debate to which even RATS is not an exclusion. A majority of authors recommend lesions $<5 \mathrm{~cm}$ for proceeding with RATS $(2,31)$. A large tumor size might have a relative contraindication with a more challenging manipulation, thus increasing the risk of an open conversion. Manipulations of large-sized tumors within the thoracic cavity may cause seeding of the tumor in the surrounding tissues and pleura. In contrast, trans-sternal operations have no size limitation. Tumor dimension might represent an important bias when comparing the open and closed surgical techniques.

\section{Early and late outcomes after RATS thymoma resections}

In a previous study, 164 patients with early-stage thymoma (Masaoka stage I and II) were operated using median sternotomy (108 patients) or the robotic approach (56 patients) (32). They were retrospectively analyzed for the duration of surgery, amount of blood loss, complications, duration of chest drainage and postoperative hospitalization, oncological results, and total costs (32). The results were analyzed after matching the propensity score. Compared with the median sternotomy, robotic thymectomy had significantly longer operative times but less intraoperative blood loss, less perioperative complications, shorter time to chest drainage removal, and shorter hospitalization duration. The median cost for the trans-sternal approach was significantly higher than the cost of the robotic procedure, mainly due to longer hospitalization (32). However, in many countries, factors involved in cost analysis could be different, thus affecting the comparison. Especially, with prevalent state insurance, trans-sternal thymoma resection 
can be much cheaper. From an oncological perspective, there were no differences between the two groups with regard to thymoma recurrence. However, the follow-up period of the trans-sternal group was significantly longer than that of the RATS thymoma resection group. The study authors thus concluded that robotic thymectomy for earlystage thymoma is a technically safe and feasible procedure with low complication rate (32).

Several authors compared trans-sternal thymoma resections with RATS thymectomy (15,16,33-35). Cakar et al. (34) published their experience after this comparison and demonstrated lower rates of complications and reduced postoperative hospitalization duration in the RATS group. Balduyck et al. (16) demonstrated that patients in the RATS group have a better postoperative recovery than those in the trans-sternal thymoma resection group. Other authors supported the previous findings with results, such as reduced blood loss and hospitalization duration following RATS thymectomy $(35,36)$. In these studies, the population was heterogeneous, which may pose a challenge when evaluating the outcomes (thymoma and/or MG, various stages of $M G$ and thymoma).

Some surgeons do not prefer the use of robotic surgery for thymoma due to the possible oncological concerns such as the rupture of the capsule with implantation of the tumor during endoscopic manipulations and the increased risk of local recurrence (due to reduced safety margins after a minimally invasive resection). Furthermore, there is a lack of long-term oncological outcomes [the longest followup period is 62 months as reported by Liu et al. (36)]. The rarity of these tumors is an additional factor that tamed the popularity of minimally invasive resection for early-stage thymomas. Recently, a systematic review and meta-analysis of the current literature regarding minimally invasive (VATS and robotic) versus open thymectomy for thymic malignancies showed no significant differences in the $\mathrm{R} 0$ resection rate and loco-regional recurrence in patients with Masaoka stages I and II tumors (37). Thymomas are low-grade tumors and have an indolent clinical course. Recurrences generally develop after 60 months of onset (38). Therefore, we believe that a follow-up of at least 10 years is essential to reach a solid conclusion. According to the current literature, RATS appears to be safe in oncology patients during the midterm follow-up.

In patients who had a thymoma resection, recurrences usually occur after a decade instead of 1-2 years. Therefore, careful consideration is essential while establishing outcomes of minimally and before extending the technical boundaries using invasive approaches in straightforward cases.

\section{Parathyroid adenoma}

Parathyroid glands are ectopic in approximately $15-20 \%$ of the cases (39), and when they become hyperfunctional, they can lead to hyperparathyroidism. In such cases, minimally invasive thoracic surgery has been proposed as an alternative to the traditional open approach with an expectation of less morbidity and improved outcomes. Surgical resection may be feasible through a cervical incision (40). Of these patients, $1-2 \%$ might need a transthoracic approach owing to a mediastinal parathyroid gland located in the deep mediastinum. These lesions are traditionally treated with open surgery. In a study by Mayo Clinic, primary hyperparathyroidism was reported as the main diagnosis in 32 patients (97\%); technetiumsestamibi contrast scan was used in 23 patients $(70 \%)$ for preoperative localization of lesions. In this study, minimally invasive resections were performed in 18 patients $(55 \%)$, and 15 patients $(45 \%)$ underwent open surgery. Minimally invasive surgical technique, including VATS, was performed in nine patients $(27 \%)$ and robotic approach in one patient. Open surgeries are performed by the transcervical, mediastinal, or manubrial split approach. The most common opensurgery approach was median sternotomy, which was performed in 11 patients (33\%). Parathyroid hormone was intraoperatively monitored in 22 patients $(67 \%)$. The ectopic glands were found to be in the thymus in 15 $(45 \%)$, in the aortopulmonary window in seven $(21 \%)$, and in other intrathoracic locations in the remaining 11 patients (33\%). Parathyroid adenomas were identified in 21 patients $(64 \%)$, whereas parathyroid hyperplasia and carcinoma were identified in nine $(27 \%)$ and three $(9 \%)$ patients, respectively. The most common complication was hypocalcemia in four patients $(12 \%)$. The mean hospitalization duration was significantly shorter in the minimally invasive surgery group ( 2 vs. 6 days; $\mathrm{P}<0.001$ ); however, mortality and morbidity were not statistically different between the two groups. According to this study, the robotic approach was reported to be very uncommon for treating parathyroid adenoma in this series (41). In contrast, Ismail et al. reported feasibility of RATS for resection of ectopic parathyroid glands in obscure and tight anatomical locations, such as the mediastinum (42). Furthermore, Ward et al. described a series of five robot- 


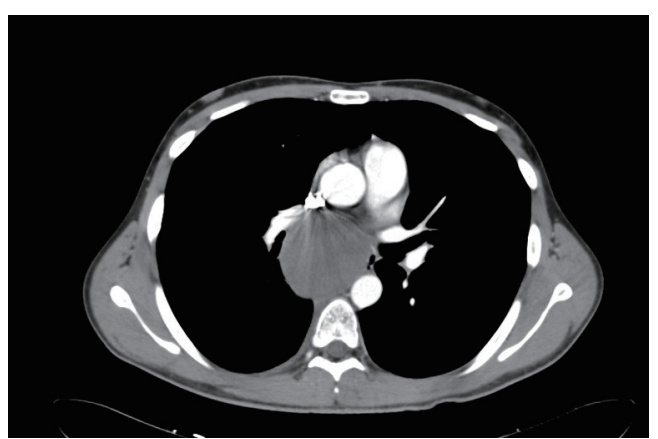

Figure 8 One of our bronchogenic cysts patient at age 18 with severe chest pain and fever. He underwent urgent operation.

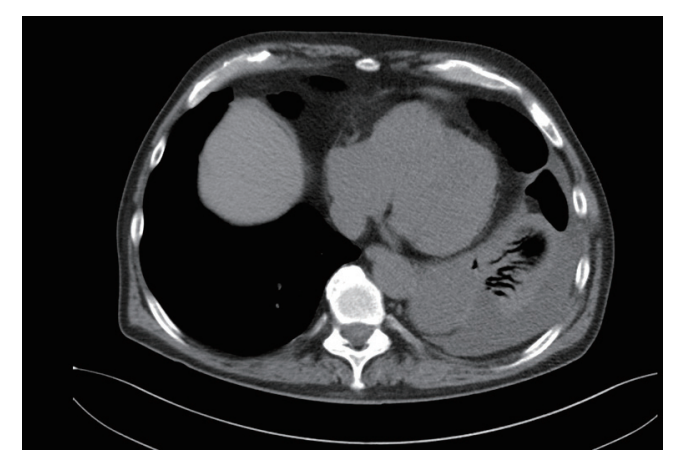

Figure 9 Ampiyema and bronchogenic cysts. The patient underwent robotic bronchogenic cyst excision and decortication. He was having routine hemodialysis.

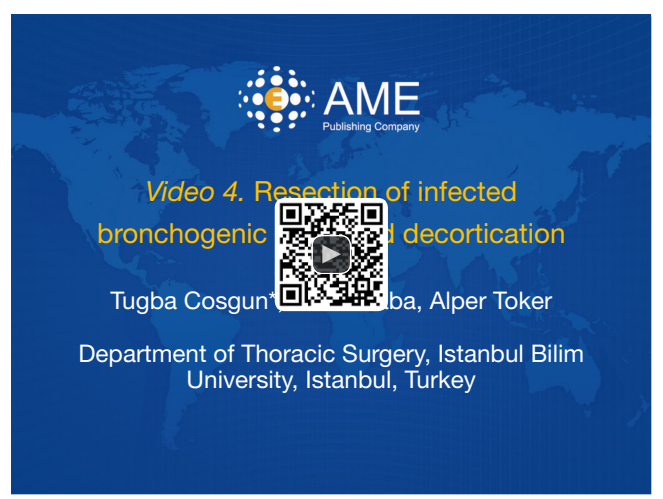

Figure 10 Resection of infected bronchogenic cysts and decortication (49).

Available online: http://www.asvide.com/article/view/28635

assisted complete resections with thymectomy in patients with mediastinal ectopic parathyroid adenomas with no complication, morbidity, and mortality (43).

\section{Middle mediastinum}

\section{Bronchogenic cysts}

Bronchogenic cysts can be either intraparenchymal or intramediastinal. Mediastinal type of cysts can compress the neighboring organs, resulting in symptoms such as wheezing, coughing, dyspnea, atelectasis, and dysphagia. Occasionally, bloody expectoration, bloody sputum, hemoptysis, fever, and pyothorax arise (44) (Figure 8). However, most of the cases are diagnosed incidentally following imaging for other reasons (45).

The open surgical approach was the routine surgical approach for complete cyst removal. Subsequently, VATS was preferred because it was less invasive than the open approach. Nonetheless, VATS has disadvantages while manipulating in narrow spaces, such as the upper and lower thoracic spaces (near the apex or diaphragm), immediately adjacent to the vertebrae and aorta (44).

Due to dense adhesions between cysts and neighboring organs, surgery via thoracotomy can pose some complications. However, RATS can prevent incomplete resection of cysts or complications because of its threedimensional visualization, better maneuverability, and articulating instrumentation (46-48). Previously, we also demonstrated safety and feasibility for resection of bronchogenic cysts using RATS in pediatric surgery (47). Our experience also demonstrated the use of robotic surgery in resection of bronchogenic cyst in patients with concomitant infectious diseases (Figures 9,10).

\section{Pericardial cysts}

Small, asymptomatic pericardial cysts can be managed nonoperatively with observation. However, large or symptomatic cysts should be surgically resected. Robotic resection of pericardial cysts has several advantages over conventional VATS. Bacchetta et al. described its advantages because of the use of Endowrist ${ }^{\mathrm{TM}}$ technology that allows for incorporation of articulating instruments. This technology provides six degrees of freedom, enabling adequate suturing and knot tying (50).

\section{Posterior mediastinum}

Many authors reported feasibility of RATS for anterior mediastinal lesions and thymoma. Few studies report the use of RATS for posterior mediastinal lesions (46-48,50,51). Benign neurogenic tumor is the most 


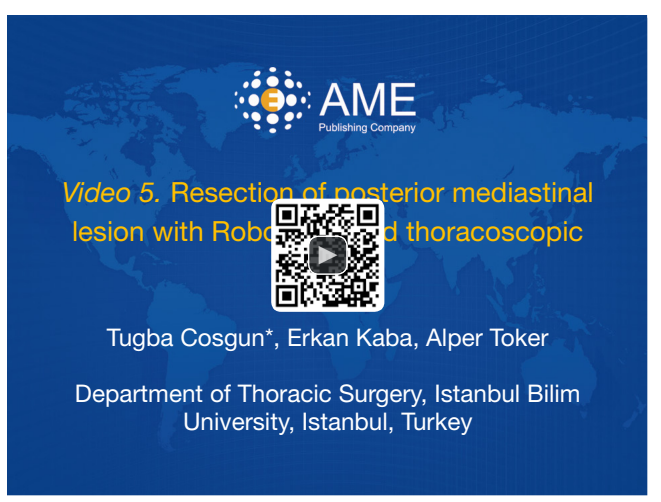

Figure 11 Resection of posterior mediastinal lesion with Robot assisted thoracoscopic surgery (57).

Available online: http://www.asvide.com/article/view/28636

common type of lesions in this category and is usually asymptomatic and diagnosed incidentally. Usually, thoracic neurogenic tumors are benign lesions that can originate from any nervous structure derived from the cells of the nerve sheath, autonomic ganglia, paraganglia, intercostal, sympathetic, or vagus nerve (51-55). These tumors are found in the thoracic cavity, mainly located in the paravertebral sulcus region or posterior mediastinum. Of all mediastinal tumors, $12-20 \%$ originate from the neural tissue. Generally, they develop asymptomatically and are found incidentally. Of all intrathoracic neurogenic tumors, $75-95 \%$ are located in the posterior mediastinum. Schwannomas and neurofibromas develop from the nerve sheath, ganglioneuromas and neuroblastomas arise from the autonomic ganglions, and paragangliomas and pheochromocytomas develop from the paraganglion. Generally, they arise from intercostal nerve rami or the sympathetic nerve region. Malignancy rate for these tumors is $6 \%$ in adults and $40 \%$ in children during the first 5 years of life. Ten percent of these have an extension into the neurogenic canal and are named "dumbbell tumors". Approximately $14 \%$ of thoracic neurogenic tumors are associated with von Recklinghausen neurofibromatosis, and $50 \%$ of the adult patients with malignant tumors have this disease. Posterolateral thoracotomy has been the standard approach for the surgical excision of these lesions. However, in the past 15 years, VATS has been advocated as a safe and effective method for the resection of such tumors. There are several contraindications to VATS, which include a large tumor size $(>6 \mathrm{~cm})$, intraspinal extension, spinal artery involvement, previous thoracic surgery, apical location at the chest (located close to the satellite ganglion and proximity to great vessels), because they carry an increased risk of peri-postoperative complications. Feasibility and contraindications of VATS have been largely discussed by other authors (56).

A surgical technique with either VATS or RATS, involves incising the parietal pleura over the mass using electrocautery or other coagulation tools like LigaSure (Covidien, Minneapolis, Minnesota, USA), harmonic scalpel (Ethicon, New Jersey, USA), or bipolar Maryland forceps (Intuitive Surgical, Inc., Sunnyvale, CA, USA). Blunt dissection is carefully performed considering the intercostal and vertebral vessels, which are divided by clipping if needed. After securing the feeding vessels, the tumor is released from the neural tissue, is removed from the body, and is placed in a plastic bag (Figure 11). A chest drain is inserted from the camera port after injecting intercostal analgesics. Conversion to thoracotomy may be necessary in cases of excessive bleeding or inability to perform the procedure. Thus, it is recommended to use a chest retractor during the early steps of the procedure. Intracapsular resection using VATS has been proposed for tumors located at the apex, around the T2 and T3 nerve root near large arteries, veins, and staller ganglion. However, intracapsular resection is not recommended by some authors, and open surgery has been recommended instead of intracapsular resection. Neurosurgeons perform posterior hemilaminectomy for "dumbbell tumors". In this procedure, the patients are positioned prone and a posterior midline incision is made; the posterior hemilaminectomy is then completed at the corresponding level. When the tumor is totally extradural, the originating nerve root is divided by ligation. Tumors with intradural extension need additional care and surgeons experienced in spinal surgery. For such tumors, the dura is opened, and the tumor is dissected from the spinal cord. After the intraspinal component of the tumor is released, it is pushed into the intrathoracic cavity to complete the procedure in a posterolateral thoracotomy position, preferably with VATS (56).

For neurogenic tumors, the complications associated with posterior mediastinal mass resection are observed in 20 $30 \%$ of cases. These complications include radicular pain, atelectasis, phrenic nerve palsy, and Horner's syndrome. Our group believes that conversion to open surgery is not a complication but rather a technical step needed in oncology patients. Conversion is reported in $22-30 \%$ of patients and is attributed to an intraspinal extension, largeness, pleuro 


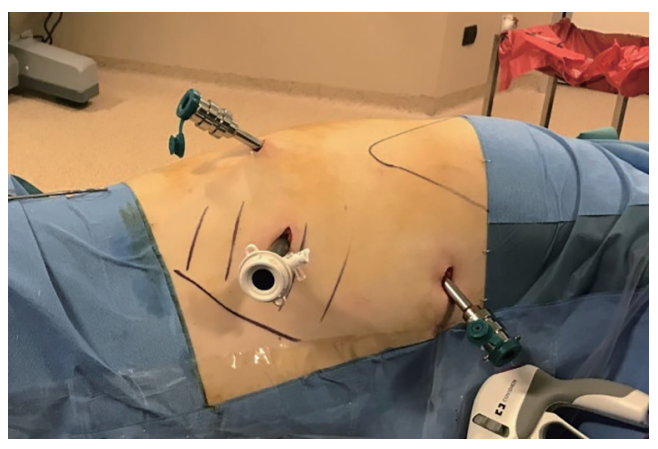

Figure 12 Positions of ports for approach for lesion located in posterior mediastinal lesions.

parenchymal adhesions, and difficult tumor locations. Other posterior mediastinal lesions include cysts, esophageal tumors, lymphadenopathies, lesions from infections, and inflammatory lesions (54).

\section{Operative approach to posterior mediastinum with robotic surgery}

Zirafa $e t a l$. has reported a four-arm technique where the camera port and two posterior ports are placed in the seventh or eighth intercostal space and one anterior port is placed in the fifth or sixth intercostal space in anterior axillary line (51). The author also emphasizes that $\mathrm{CO}_{2}$ insufflation $(5-8 \mathrm{mmHg})$ can be used to increase the space and that grasper can be used to retract the lung for better exposure (51).

We preferred the three-port, robot-assisted approach in all our cases. We placed a 30 degrees camera port in the eighth intercostal space in the posterior axillary line. With guidance from this port, we placed the anterior arm in the fifth intercostal space in the anterior axillary line and the posterior arm in the eighth intercostal space, $5-6 \mathrm{~cm}$ posterior to the camera port (Figure 12).

Cerfolio described their experience regarding this issue and reported low morbidity rate and no mortality (46). Kajiwara et al. presented three extended cases that were locally adjacent to the vertebrae or aorta and demonstrated that RATS is also a safe method in these cases (58).

\section{Esophageal lesions}

Leiomyoma is one of the most common benign esophageal neoplasms, accounting for more than $50 \%$ of these tumors (59). Different invasive surgical methods have been described for management of such lesions. Enucleation of a 4-cm leiomyoma via RATS has been described by Khalaileh et al. (60). Successful resection of esophageal cysts with RATS has also been reported owing to the articulating instrumentation and 3-dimensional magnified view that provide an advantage over the standard VATS approach (61).

\section{Acknowledgements}

None.

\section{Footnote}

Conflicts of Interest: The authors have no conflicts of interest to declare.

Informed Consent: Written informed consent was obtained from the patient for publication of this manuscript and any accompanying images.

\section{References}

1. Toker A, Tanju S, Sungur Z, et al. Videothoracoscopic thymectomy for nonthymomatous myasthenia gravis: results of 90 patients. Surg Endosc 2008;22:912-6.

2. Toker A, Erus S, Ozkan B, et al. Does a relationship exist between the number of thoracoscopic thymectomies performed and the learning curve for thoracoscopic resection of thymoma in patients with myasthenia gravis? Interact Cardiovasc Thorac Surg 2011;12:152-5.

3. Ye B, Tantai JC, Ge XX, et al. Surgical techniques for early stage thymoma: video assisted thoracoscopic thymectomy versus transsternal thymectomy. J Thorac Cardiovasc Surg 2014;147:1599-603.

4. Manoly I, Whistance RN, Sreekumar R, et al. Early and mid-term outcomes of trans-sternal and video-assisted thoracoscopic surgery for thymoma. Eur J Cardiothorac Surg 2014;45:e187-93.

5. Toker A. Standardized definitions and policies of minimally invasive thymoma resection. Annals of Cardiothoracic Surgery. Ann Cardiothorac Surg 2015;4:535-9.

6. Toker A, Sonett J, Zielinski M, et al. Standard terms, definitions and policies for minimally invasive resection of thymoma. J Thorac Oncol 2011;6:S1739-42.

7. Wolfe GI, Kaminski HJ, Sonnett JR, et al. Randomized trial of thymectomy in myasthenia gravis. J Thorac Dis 2016;8:E1782-3.

8. Rückert JC, Ismail M, Swierzy M, et al. Thoracoscopic 
thymectomy with the da Vinci robotic system for myasthenia gravis. Ann N Y Acad Sci 2008;1132:329-35.

9. Moorthy K, Munz Y, Dosis A, et al. Dexterity enhancement with robotic surgery. Surg Endosc 2004;18:790-5.

10. Kaba E, Cosgun T, Ayalp K, et al. Robotic thymectomya new approach for thymus. J Vis Surg 2017:8;3:67.

11. Rückert JC, Swierzy M, Ismail M. Comparison of robotic and nonrobotic thoracoscopic thymectomy: A cohort study. J Thorac Cardiovasc Surg 2011;141:673-7.

12. Keijzers M, de Baets M, Hochstenbag M, et al. Robotic thymectomy in patients with myasthenia gravis: neurological and surgical outcomes. Eur J Cardiothorac Surg 2015;48:40-5.

13. Fok M, Bashir M, Harky A, et al. Video-assisted thoracoscopic versus Robotic-assisted thoracoscopic thymectomy: systematic review and meta-analysis. Innovations (Phila) 2017;12:259-64.

14. Buentzel J, Heinz J, Hinterthaner M, et al. Robotic versus thoracoscopic thymectomy: The current evidence. Int J Med Robot 2017;13(4).

15. Seong YW, Kang CH, Choi JW, et al. Early clinical outcomes of robot-assisted surgery for anterior mediastinal mass: its superiority over a conventional sternotomy approach evaluated by propensity score matching. Eur J Cardiothorac Surg 2014;45:e68-73; discussion e73.

16. Balduyck B, Hendriks JM, Lauwers P, et al. Quality of life after anterior mediastinal mass resection: a prospective study comparing open with robotic-assisted thoracoscopic resection. Eur J Cardiothorac Surg 2011;39:543-8.

17. Wu L, Lin L, Liu M, et al. Subxiphoid uniportal thoracoscopic extended thymectomy. J Thorac Dis 2015;7:1658-60.

18. Kaba E, Cosgun T, Ayalp K, et al. Right sided VATS thymectomy: "current standards of extended thymectomy for myasthenia gravis. Video-assist Thorac Surg 2017;2:30.

19. Cosgun T, Kaba E, Toker A. After dissection from right sided pericardiophrenic tissue and separation of right thymus from the pericardium and superior vena cava, pulling down the right upper pole of the thymus. Asvide 2018;5:872. Available online: http://www.asvide.com/ article/view/28631

20. Cosgun T, Kaba E, Toker A. Pulling of left upper pole, visible thymic veins and using clip for vein. Asvide 2018;5:873. Available online: http://www.asvide.com/ article/view/28632

21. Mineo TC, Pompeo E, Lerut TE, et al. Thoracoscopic thymectomy in autoimmune myasthesia: results of left- sided approach. Ann Thorac Surg 2000;69:1537-41.

22. Pardolesi A, Spaggiari L. Left Side Robotic Approach for Extended Thymectomy: Surgical Technique and Preliminary Experience. Austin J Surg 2016;3:1090.

23. Elsayed HH, Gamal M, Raslan S, et al. Video-assisted thoracoscopic thymectomy for non-thymomatous myasthenia gravis: a right-sided or left-sided approach? Interact Cardiovasc Thorac Surg 2017;25:651-3.

24. Karunanantham J, Fok M, Ali JM, et al. Subxiphoid single incision thoracoscopic surgery approach for thymectomy: a case report. J Vis Surg 2017 Oct 27;3:147.

25. Weaver H, Ali JM, Jiang L, et al. Uniportal subxiphoid video-assisted thoracoscopic approach for thymectomy: a case series. J Vis Surg 2017;3:169.

26. Zielinski M, Czajkowski W, Gwozdz P, et al. J.Resection of thymomas with use of the new minimally-invasive technique of extendedthymectomy performed through the subxiphoid-right video-thoracoscopic approach with doubleelevation of the sternum. Eur J Cardiothorac Surg 2013;44:e113-9.

27. Suda T, Kaneda S, Hachimaru A, et al. Thymectomy via a subxiphoid approach: single-port and robot-assisted.J Thorac Dis 2016;8:S265-71.

28. Toker A, Erus S, Ziyade S, et al. It is feasible to operate on pathological stage 1 and 2 thymoma patients with videoassisted thoracoscopy:analysis of factors for successful resection. Surg Endosc 2013;27:1555-60.

29. Agasthian T. Can invasive thymomas be resected by videoassisted thoracoscopic surgery? Asian Cardiovasc Thorac Ann 2011;19:225-7.

30. Cosgun T, Kaba E, Toker A. Resection of non-seminomatous germ cell tumor after neoadjuvant chemotherapy, invading the left innominate vein with RATS. Asvide 2018;5:874. Available online: http://www.asvide.com/article/view/28633

31. Marulli G, Rea F, Melfi F, et al. Robotaided thoracoscopic thymectomy for early-stage thymoma: a multicenter European study. J Thorac Cardiovasc Surg 2012;144:1125-30.

32. Marulli G, Comacchio GM, Schiavon M, et al. Comparing robotic and trans-sternal thymectomy for early stage thymoma: a propensity score-matching study. Eur J Cardiothorac Surg 2018;54:579-84.

33. Ye B, Li W, Ge XX, et al. Surgical treatment of early-stage thymomas: robot-assisted thoracoscopic surgery versus transsternal thymectomy. Surg Endosc 2014;28:122-6.

34. Cakar F, Werner P, Augustin F, et al. A comparison of outcomes after robotic open extended thymectomy for myasthenia gravis. Eur J Cardiothorac Surg 2007;31:501-4. 
35. Weksler B, Tavares J, Newhook TE, et al. Robot-assisted thymectomy is superior to transsternal thymectomy. Surg Endosc 2012; 26:261-6.

36. Liu TJ, Lin MW, Hsieh MS, et al. Videoassisted thoracoscopic surgical thymectomy to treat early thymoma: a comparison with the conventional transsternal approach. Ann Surg Oncol 2014;21:322-8.

37. Friedant AJ, Handorf EA, Su S, et al. Minimally invasive versus open thymectomy for thymic malignancies: systematic review and meta-analysis. J Thorac Oncol 2016;11:30-8.

38. Kimura T, Inoue $M$, Kadota $Y$, et al. The oncological feasibility and limitations of video-assisted thoracoscopic thymectomy for early-stage thymomas. Eur J Cardiothorac Surg 2013;44:e214-8.

39. Soler R, Bargiela A, Cordido F, et al. MRI of mediastinal parathyroid cystic adenoma causing hyperparathyroidism. J Comput Assist Tomogr 1996;20:166-8.

40. Kaplan EL, Yashiro T, Salti G. Primary hyperparathyroidism in the 1990s. Ann Surg 1992;215:300-17.

41. Said SM, Cassivi SD, Allen MS, et al. Minimally invasive resection for mediastinal ectopic parathyroid glands. Ann Thorac Surg 2013;96:1229-33.

42. Ismail M, Maza S, Swierzy M, et al. Resection of ectopic mediastinal parathyroid glands with the da Vinci robotic system. Br J Surg 2010;97:337-43.

43. Ward AF, Lee T, Ogilvie JB, et al. Robot-assisted complete thymectomy for mediastinal ectopic parathyroid adenomas in primary hyperparathyroidism. J Robot Surg 2017;11:163-169.

44. Toker A, Ozyurtkan MO, Kaba E, et al. Da Vinci Robotic System in the surgery for mediastinal bronchogenic cyst: a report on five patients. J Vis Surg 2015;1:23.

45. De Giacomo T, Diso D, Anile M, et al. Thoracoscopic resection of mediastinal bronchogenic cysts in adults. Eur J Cardiothorac Surg 2009;36:357-9.

46. Cerfolio RJ, Bryant AS, Minnich DJ. Operative techniques in robotic thoracic surgery for inferior or posterior mediastinal pathology. J Thorac Cardiovasc Surg 2012;143:1138-43.

47. Toker A, Ayalp K, Grusina-Ujumaza J, et al. Resection of a bronchogenic cyst in the first decade of life with robotic surgery. Interact Cardiovasc Thorac Surg 2014;19:321-3.

48. Kajiwara N, Taira M, Yoshida K, et al. Early experience using the da Vinci Surgical System for the treatment of mediastinal tumors. Gen Thorac Cardiovasc Surg 2011;59:693-8.

49. Cosgun T, Kaba E, Toker A. Resection of infected bronchogenic cysts and decortication. Asvide 2018;5:875. Available online: http://www.asvide.com/article/ view/28635

50. Bacchetta MD, Korst RJ, Altorki NK, et al. Resection of a Symptomatic Pericardial Cyst Using the ComputerEnhanced Da Vinci ${ }^{\mathrm{TM}}$ Surgical System. Ann Thorac Surg 2003;75:1953-5.

51. Zirafa CC, Melfi F. Robot-assisted surgery for posterior mediastinal mass. J Thorac Dis 2017;9:4929-31.

52. Xu S, Liu B, Wang X, et al. Robotic thoracic surgery of the posterior superior mediastinal mass. Ann Transl Med 2015;3:127.

53. Pavlus JD, Carter BW, Tolley MD, et al. Imaging of Thoracic Neurogenic Tumors. AJR Am J Roentgenol 2016:207:552-61.

54. Duwe BV, Sterman DH, Musani AI. Tumors of the mediastinum. Chest 2005;128:2893-909.

55. Melfi FM, Fanucchi O, Mussi A. Minimally invasive mediastinal surgery. Ann Cardiothorac Surg 2016;5:10-7.

56. Kaba E, Alomari MR, Toker A. Open surgery for posterior mediastinal neurogenic tumors. Shanghai Chest 2017;1:15.

57. Cosgun T, Kaba E, Toker A. Resection of posterior mediastinal lesion with Robot assisted thoracoscopic surgery. Asvide 2018;5:876. Available online: http://www. asvide.com/article/view/28636

58. Kajiwara N, Kakihana M, Usuda J, et al. Extended indications for robotic surgery for posterior mediastinal tumors. Asian Cardiovasc Thorac Ann 2012;20:308-13.

59. Jang KM, Lee KS, Lee SJ, et al. The Spectrum of Benign Esophageal Lesions: Imaging Findings. Korean J Radiol 2002;3:199-210.

60. Khalaileh A, Savetsky I, Adileh M, et al. Robotic-assisted enucleation of a large lower esophageal leiomyoma and review of literature. Int J Med Robot 2013;9:253-7.

61. Fernando HC, Erdem CC, Daly B, et al. Robotic assisted thoracic surgery for resection of an esophageal cyst. Dis Esophagus 2006;19:509-11.

doi: $10.21037 /$ shc.2018.11.04

Cite this article as: Cosgun T, Kaba E, Toker A. Robotic approach for mediastinal diseases: state-of-the-art and current perspectives. Shanghai Chest 2018;2:90. 